\title{
The application of traditional Chinese medicine against the tumor immune escape
}

\author{
Jing Xu, Jingjing Zhang, Jing Wang \\ Department of Hematology and Oncology, Dongzhimen Hospital, \\ Beijing University of Chinese Medicine, Beijing, China
}

\section{INTRODUCTION}

Tumor immune escape happens when tumor cells are not detected, recognized, and killed by the body's immune system. It is often under the protection of the tumor microenvironment, which refers to a complex network composed of tumor cells, immune cells, endothelial cells, stromal cells, fibroblasts and various cytokines. By interacting with stromal cells and immune cells, or by secreting cytokines, tumor cells can get rid of the recognition and attack, and suppress the immune response of the host, so as to transform the tumor microenvironment into a microenvironment conducive to tumor survival, growth and uncontrollable proliferation, eventually causing immune escape of tumor cells. ${ }^{[1-2]}$ Among them, macrophages, dendritic cells, regulatory $\mathrm{T}$ cells, myeloid-derived suppressor cells and NK cells are the main immune regulatory cells. Traditional Chinese medicine (TCM), widely used by patients with cancers in China, can reverse the immune escape of tumor by modifying the microenvironment and enhancing the function of immune cells.

According to TCM, the occurrence of tumor is related to the deficiency of healthy $q i$ and the hyperactivity of evil $q i$ in the body. It is the major principle of TCM in the treatment of malignant tumors to strengthen the healthy $q i$ and disperse the evil $q$, tonify the spleen and resolve the dampness, clear heat and detoxify. Strengthening the healthy $q i$ and dispersing the evil $q i$ method can play an anti-tumor role by regulating immune cell mediated tumor immune escape in tumor microenvironment. On the one hand, it can promote the secretion of tumor suppressor factors by macrophages, and inhibit the formation of tumor-related macrophages. ${ }^{[3-4]}$ Epimedium koreanum Nakai polysaccharides are an important active component of Epimedium. It can promote secretion of cytokines such as IFN- $\gamma$ and TNF- $\alpha$, and activate CD $4+\mathrm{T}$ cells differentiation to enhance the antitumor effect of the body. ${ }^{[5]}$ On the other hand, it can suppress the effect of myeloid derived suppressor cells (MDSCs) and reduce the angiogenesis of tumors. MDSCs are abundantly present in the tumor microenvironment, and are an important cell population that causes tumor immune escape and has immunosuppressive effects. ${ }^{[6-7]}$ By examining the level of MDSCs in peripheral blood of 31 breast cancer patients, it was found that the level of MDSCs in peripheral blood was closely related to disease progression. Shuganjianpi formula alone or combined with gemcitabine chemotherapy could inhibit the proliferation of MDSC cells and improve the immunity of the body. ${ }^{[8-9]}$

Many studies have repeatedly proven the key anti-cancer mechanism of TCM lies in regulating immune cells and mediating tumor immune escape. Pang et al..$^{[10]}$ studied the mechanism of Bufei Decoction against Non-Small Cell Lung Cancer (NSCLC) and found that tumor-associated macrophages was essential in the progression of NSCLC and were related to tumor cell immune suppression. Su et al. ${ }^{[11]}$ established a mouse 
4T1 breast cancer model and found that Xihuang Pill, a patent Chinese medicine of heat clearing and detoxification function, could promote apoptosis of Treg cells in the tumor microenvironment. Another study pointed out that Astragalus polysaccharide (APS), main component of qi tonifying herb Astragalus membranaceus, inhibited the expression of Foxp3 mRNA through cytokine and matrix repair, hampered SDF-1's recruitment of Treg cells in the micro-environment of liver cancer, and reduces the immunosuppressive effect of Treg cells. ${ }^{[12]}$ Other research of Wolfberry, Scutellaria barbata, and Coix lacryma-jobi also showed their different properties to improve antitumor immunity. ${ }^{[13-15]}$

The wisdom of TCM method is in symphony with the most recent understanding of anti-cancer strategy. A comprehensive database of TCM on immuno-oncology has been established based on the chemical ingredients of herbs that interact with the immune-oncology targets. ${ }^{[16]}$ It provides us a new tool for potential herbs with immune regulating properties, yet all results need to be confirmed with further experiments and trials. Up to now, the scientific explanation of TCM for cancer is still rather preliminary. It calls for more rigorous experiments and clinical trials in order to decipher the capacity of TCM in the anti-tumor immunity.

\section{Source of Funding}

This work was supported by the Young Scientist Fund (81503575) and Key Program (81630080) of National Natural Science Foundation of China, the Golden Bridge Project of Beijing Association for Science and Technology (ZZ20059) and the Elite Program of Dongcheng district of Beijing (2020-dchrcpyzz-38).

\section{Conflict of Interest}

None declared.

\section{Acknowledgement}

We thank Elizabeth Gullen from Yale University for the critical reading of this article.

\section{REFERENCES}

1. Wu T, Dai Y. Tumor microenvironment and therapeutic response. Cancer Lett 2017; 387: 61-8.

2. Ryu D, Kim SJ, Hong Y, Jo A, Kim N, Kim HJ, et al. Alterations in the Transcriptional Programs of Myeloma Cells and the Microenvironment during Extramedullary Progression Affect Proliferation and Immune Evasion. Clin Cancer Res 2020; 26: 935-44.
3. Park JY, Sung JY, Lee J, Park YK, Kim YW, Kim GY, et al. Polarized CD163+ tumor-associated macrophages are associated with increased angiogenesis and CXCL12 expression in gastric cancer. Clin Res Hepatol Gastroenterol 2016; 40: 357-65.

4. Yuri P, Hendri AZ, Danarto R. Association between tumor-associated macrophages and microvessel density on prostate cancer progression. Prostate Int 2015; 3: 93-8.

5. Wang C, Feng L, Su J, Cui L, Dan Liu, Yan J, et al. Polysaccharides from Epimedium koreanum Nakai with immunomodulatory activity and inhibitory effect on tumor growth in LLC-bearing mice. J Ethnopharmacol 2017; 207: 8-18.

6. Zhou J, Wu J, Chen X, Fortenbery N, Eksioglu E, Kodumudi KN, et al. Icariin and its derivative, ICT, exert anti-inflammatory, anti-tumor effects, and modulate myeloid derived suppressive cells (MDSCs) functions. Int Immunopharmacol 2011; 11: 890-8.

7. Khaled YS, Ammori BJ, ElkordE. Myeloid-derived suppressor cells in cancer: recent progress and prospects. Immunol Cell Biol 2013; 91 : 493-502.

8. Lu Y, Li J, Qi X, Pei Y, Shi W, Lin H. Effects of Shugan Jianpi Formula on myeloid-derived suppression cells-mediated depression breast cancer mice. Chin J Integrat Med 2017; 23: 453-60.

9. Lv YT. Effect of Depression on MDSC-induced Immune Remodeling in Breast Cancer and Intervention of Shuganjianpi formula. Beijing: Beijing University of Chinese Medicine, 2013. [Master Thesis]

10. Pang L, Han S, Jiao Y, Jiang S, He X, Li P. Bu Fei Decoction attenuates the tumor associated macrophage stimulated proliferation, migration, invasion and immunosuppression of non-small cell lung cancer, partially via IL-10 and PD-L1 regulation. Int J Oncol 2017; 51: 25-38.

11. Su L, Jiang Y, Xu Y, Li X, Gao W, Xu C, et al. Xihuang pill promotes apoptosis of Treg cells in the tumor microenvironment in 4T1 mouse breast cancer by upregulating MEKK1/SEK1/JNK1/AP-1 pathway. Biomed Pharmacother 2018; 102: 1111-9.

12. Liu QY, Yao YM, Yu Y, Dong N, Sheng ZY. Astragalus polysaccharides attenuate postburn sepsis via inhibiting negative immunoregulation of CD4+ CD25(high) T cells. PLoS One 2011; 6: e19811.

13. He YL, Ying Y, Xu YL, Su JF, Luo H, Wang HF. [Effects of Lycium barbarum polysaccharide on tumor microenvironment T-lymphocyte subsets and dendritic cells in H22-bearing mice.] J Chin Integrat Med = Zhongxiyi Jiehe Xuebao 2005; 3: 374-7.

14. Kan X, Zhang W, You R, Niu Y, Guo J, Xue J. Scutellaria barbata D. Don extract inhibits the tumor growth through down-regulating of Treg cells and manipulating Th1/Th17 immune response in hepatoma H22-bearing mice. BMC Complement Altern Med 2017; 17: 41.

15. Yang Y, Li D, Ding X, Xu W, Zheng Q. Norcantharidin combined with Coix seed oil synergistically induces apoptosis and inhibits hepatocellular carcinoma growth by downregulating regulatory $\mathrm{T}$ cells accumulation. Sci Rep 2017; 7: 9373.

16. Liu Z, Cai C, Du J, Liu B, Cui L, Fan X, et al. TCMIO: A Comprehensive Database of Traditional Chinese Medicine on Immuno-Oncology. Front Pharmacol 2020; 11: 439.

How to cite this article: $\mathrm{Xu} \mathrm{J}$, Zhang J, Wang J. The application of traditional Chinese medicine against the tumor immune escape. J Transl Intern Med 2020; 8: 203-4. 